\title{
Comparison between headfirst and tail-first direction of sperm for intracytoplasmic sperm injection
}

\author{
Pallop Pongsuthirak*
}

Department of Obstetrics and Gynecology, Buddhachinaraj Hospital Medical School, Phitsanulok 65000, Thailand

Received: 30 September 2019

Accepted: 07 October 2019

\section{*Correspondence:}

Dr. Pallop Pongsuthirak,

E-mail: palloppong@yahoo.co.th

Copyright: () the author(s), publisher and licensee Medip Academy. This is an open-access article distributed under the terms of the Creative Commons Attribution Non-Commercial License, which permits unrestricted non-commercial use, distribution, and reproduction in any medium, provided the original work is properly cited.

\begin{abstract}
Background: Intracytoplasmic sperm injection (ICSI) is used for assisted fertilization. Sperm can be injected headfirst and tail-first. This study aimed to investigate the effect of the headfirst or tail-first injection of sperm into the cytoplasm during ICSI on oocyte survival, fertilization, blastocyst development, implantation, and clinical pregnancy. Methods: A prospective study was carried out by enrolling 79 women who had undergone IVF with male factor infertility between September 2016 and June 2019. The sibling oocytes were randomly inseminated by headfirst ICSI (405 oocytes) and tail-first ICSI (393 oocytes). After fertilization, the embryos were cultured until blastocyst and single embryos were transferred under ultrasound guidance.

Results: There was no difference in survival rates $(98.8 \%$ versus $98.9 \% ; \mathrm{p}=0.551)$, fertilization rate $(71.5 \%$ versus $74.8 \% ; \mathrm{p}=0.333$ ), and formation of blastocysts $(50.7 \%$ versus $51.2 \% ; \mathrm{p}=0.970)$ between headfirst and tail-first direction of sperm during ICSI. Implantation and clinical pregnancy were also not significantly different.

Conclusions: The outcomes showed that the direction of sperm injection (headfirst and tail-first) had no significant effect on oocyte survival, fertilization, blastocyst development, implantation, and clinical pregnancy.
\end{abstract}

Keywords: Developmental competence, Headfirst, Intracytoplasmic sperm injection, Male infertility, Sperm direction, Tail-first

\section{INTRODUCTION}

Fertilization composes of a cascade of interactions between the cumulus-oocyte complex and sperm. Naturally, there is a need for sperm capacitation, binding, and penetration of the zona pellucida, passage through the perivitelline space, binding and fusion to the oolemma, activation of the cortical reaction and formation of the male pronucleus. ${ }^{1,2}$ In male infertility, the sperm is unable to fertilize the oocyte successfully due to defects in any step. ${ }^{3}$ Nevertheless, intracytoplasmic sperm injection (ICSI) can overcome such conditions by direct injection of single sperm into the cytoplasm of the oocyte. ${ }^{4}$ Traditionally, the sperm's head is the leading part to be injected first with the notion that it might mimic normal fertilization. In some instances, however, sperm can be inserted tail-first during the procedure. The impact of the direction of sperm on fertilization and embryonic development from ICSI is not yet clearly understood. This study aimed to evaluate the effect of the sperm direction (headfirst and tail-first) during ICSI on oocyte survival, fertilization, blastocyst development, implantation, and clinical pregnancy.

\section{METHODS}

The Research Ethics Committee of Buddhachinaraj Hospital Medical School (No. 34/2562) and Thai Clinical 
Trial Registration (TCTR 20190826004) approved this study. The prospective randomized clinical trial was conducted from September 2016 to June 2019 on the patients who underwent IVF treatment. They were invited to participate in the study and signed written informed consent.

\section{Inclusion criteria}

- $\quad$ The patients aged 20-35 years old.

- Their husband had male factor infertility according to the WHO criteria (2010), volume $<1.5 \mathrm{ml}$, concentration $<15 \times 106 / \mathrm{ml}$, total sperm count $<39 \times 106 / \mathrm{ml}$, motility $<28 \%$ progressive, vitality $<59 \%$ live, morphology $<3 \%$ normal, and needed ICSI for assisted fertilization.

- The number of retrieved oocytes were more than 6 mature oocytes.

\section{Exclusion criteria}

- The patient could not have fresh embryo transfer from any cause.

- The patient cannot tolerate medication during luteal support.

The oocytes were randomly allocated into two groups; namely headfirst direction (Group I) and tail-first direction (Group II). The oocytes were denuded of their cumulus cells 3 hours after retrieval by a 30 -second exposure to $80 \mathrm{IU}$ hyaluronidase in a flushing medium (Medi-Cult, Beemem, Belgium) with the use of a denuding pipette (Flexipet, Cook). Those oocytes which had extruded their first polar bodies were inseminated by intracytoplasmic sperm injection (ICSI). Embryo transfers were selected randomly depending on whether the transferred embryos originated from the headfirst or tail-first ICSI group.

\section{Controlled ovarian hyperstimulation and oocyte retrieval}

All patients were treated by controlled ovarian hyperstimulation (COH). Briefly, a gonadotropinreleasing hormone agonist was administered for downregulation in the mid-luteal phase of the previous cycle. Follicle-stimulating hormones and/or human menopausal gonadotropins in individually adapted doses were injected after pituitary desensitization. The patients then received 5000-10,000 IU of human chorionic gonadotropin (hCG) when at least three follicles were $\geq 18 \mathrm{~mm}$. Oocyte retrieval was performed under transvaginal ultrasound guidance 36-38 hours later.

\section{Sperm preparation and insemination}

Semen samples were collected by masturbation on the morning of the oocyte retrieval day following 3-5 days of sexual abstinence. Sperm concentration, motility, and morphology were evaluated under a light microscope based on the World Health Organization criteria (WHO, 2010). Sperm were prepared by Sil-select Plus (Beemem, Belgium) gradient centrifugation at $300 \mathrm{~g}$ for 20 minutes. The sperm pellet was washed twice with $2 \mathrm{ml}$ of flushing medium at $200 \mathrm{~g}$. The sperm pellet was then resuspended in fresh medium and incubated at $37^{\circ} \mathrm{C}$ in a $6 \% \mathrm{CO}_{2}$ incubator waiting for injection.

\section{Intracytoplasmic sperm injection (ICSI)}

The ICSI procedure was performed using an Olympus CK2 inverted microscope (Olympus, London, UK) under Hoffman modulation optics (Modulation Optics Inc., Greenvale, NY) on the heated stage. Holding and injection pipettes were commercially available (Cook, Australia). The sperm suspension was placed in a $10 \mu \mathrm{l}$ droplet of $10 \%$ polyvinyl-pyrrolidone (Medicult) at the 3 o'clock position just before the ICSI procedure. Injection of the oocyte was performed in microdroplets of HEPES buffered IVF medium (Sperm Preparation Medium; Medicult, Jyllinge, Denmark) in Falcon 1006 dishes (Fahrenheit, Rotherham, South Yorkshire, UK) under paraffin oil (Cook, Australia). A normal morphological motile sperm that had migrated to the 9 o'clock position was selected and immobilized by crushing its tail with the injection micropipette and then aspirated tail first into the pipette. Oocytes were randomly allocated to headfirst and tail-first groups. The oocyte to be injected was secured with the holding pipette at the 9 o'clock position adjacent to the polar body (12 o'clock position). The micropipette containing the sperm was then trespassed through the zona pellucida and oolemma into the ooplasm at the 3 o'clock position of the oocyte. Perforation of the oolemma was ensured by the free flow suction of cytoplasm into the micropipette and the sperm was then slowly released head first and placed at the same 9 o'clock position within the cytoplasm. The pipette was then withdrawn gently, and the oocyte was detached from the holding pipette.

In a tail-first injection, the sperm was aspirated head first into the pipette after immobilization and the tail of sperm was injected first into ooplasm in a similar manner. Preparing time defined as the time since the sperm was immobilized and completely aspirated into the micropipette was recorded. The same ICSI practitioner performed all micromanipulations by using the same micromanipulation equipment to minimize interoperator variation. Following ICSI, oocytes were cultured in groups of up to three in $20 \mu 1$ drops of cleavage medium in a tissue culture dish covered with paraffin oil.

\section{Assessment of fertilization and embryo development}

Fertilization was assessed 18 hours after ICSI for the presence of two pronuclei and culturing was continued for a further 48 hours. The embryos were transferred into a blastocyst medium (Cook, Australia), and cultured for another 48 hours. On day 5, embryo development was 
classified at the blastocyst stage. Grade 1 blastocysts had good cellular development of both the inner cell mass (ICM) and trophectoderm (TE), grade 2 blastocysts had average development of the ICM and TE, while grade 3 had poor cellular development.

\section{Embryo transfer and pregnancy test}

One blastocyst was chosen for transfer according to the top morphological grade under ultrasound guidance. Pregnancy was established by presenting $\beta$-hCG at $9-10$ days after embryo transfer and then monitored serially for the rising titer. Implantation was documented later by the appearance of the gestational sac in the uterus. A clinical pregnancy was considered if a fetal heartbeat was detected. The supernumerary embryos with good quality were vitrified for future treatment.

\section{Statistical analysis}

All analyses were performed using SPSS software (version 13.0, SPSS Inc., Chicago, IL). Continuous data were compared using Student's t-test and proportional data were compared using the $\chi 2$ test. A p-value of $<0.05$ was considered statistically significant. Our sample size calculation showed that at least 363 oocytes in each arm would be required to demonstrate a $10 \%$ difference in fertilization rates between oocytes in the two groups, given a type I error of 5\% (two-tailed) and a type II error of $20 \%$.

\section{RESULTS}

A total of 79 women aged $31.4 \pm 2.5$ years old were eligible in the study. Twenty-nine cases $(36.7 \%)$ had tubal factor infertility, $12(15.2 \%)$ had endometriosis, 18 $(22.8 \%)$ had polycystic ovary syndrome, and $20(25.3 \%)$ had unexplained infertility (Table 1). Seven hundred and ninety-eight mature oocytes were collected. The sibling oocytes were randomly assigned to headfirst ICSI (Group I) 405 oocytes and tail-first ICSI (Group II) 393 oocytes. There were no significant differences in all treatment parameters between the two groups. The details of fertilization and embryo development were summarized in Table 2. No significant difference was observed in fertilization and blastocyst rate but preparing time for headfirst ICSI is longer than tail-first ICSI $(12.4 \pm 2.5$ versus $9.2 \pm 2.1 \mathrm{~min}, \mathrm{p}<0.001)$.

The embryo transfers were conducted in 79 cycles, of which 39 cycles obtained the embryos from group I and 40 cycles from group II. Implantation rates and clinical pregnancies were not significantly different between the patients from the two groups (Table 2).

Table 1: Demographic characteristics of women in the study.

\begin{tabular}{|ll|}
\hline Characteristics & $\mathrm{n}(\%)$ \\
\hline Patients & 79 \\
\hline Age years (Mean \pm SD) & $31.4 \pm 2.5$ \\
\hline Cause of infertility & \\
\hline Tubal & $29(36.7 \%)$ \\
\hline Endometriosis & $12(15.2 \%)$ \\
\hline PCOS & $18(22.8 \%)$ \\
\hline Unexplained & $20(25.3 \%)$ \\
\hline
\end{tabular}

Table 2: Comparison of outcomes between headfirst and tail-first ICSI.

\begin{tabular}{|llll|}
\hline & Headfirst ICSI & Tail-first ICSI & P-value \\
\hline Oocytes & 405 & 393 & 0.551 \\
\hline Surviving oocytes & $400(98.8 \%)$ & $389(98.9 \%)$ & 0.333 \\
\hline Fertilized oocytes & $286(71.5 \%)$ & $291(74.8 \%)$ & 0.970 \\
\hline Blastocyst & $145(50.7 \%)$ & $149(51.20)$ & 0.932 \\
\hline Implantation & $14 / 39(35.9 \%)$ & $15 / 40(37.5 \%)$ & 0.935 \\
\hline Clinical pregnancy & $13 / 39(33.3 \%)$ & $13 / 40(32.5 \%)$ & \\
\hline
\end{tabular}

$*^{*}$ 2-tests.

\section{DISCUSSION}

Intracytoplasmic sperm injection (ICSI) is generally advocated for assisted fertilization in male factor infertility. It skips over the truncated processes of sperm and oocyte interaction. Mostly, sperm was injected headfirst to mimic natural fertilization but tail-first could be performed with successful fertilization. ${ }^{3,4}$ This study found no statistical difference in sperm direction during ICSI on oocyte survival, fertilization, blastocyst 
development, implantation, and clinical pregnancy. The results go in line with previous reports. Suh et al, showed no significant difference in fertilization rates of headfirst and tail-first injection $(81.8 \%$ versus $72.7 \%){ }^{5}$ Subsequently, Woodward et al, demonstrated a fertilization rate of $71.9 \%$ in headfirst ICSI and $76.6 \%$ in tail-first ICSI that was not statistically different. ${ }^{6}$ In a recent study, Hiraoka et al, also found a similar fertilization rate of headfirst and tail-first piezo-ICSI ( $86.7 \%$ versus $90.9 \%$, respectively). ${ }^{7}$

ICSI is not a natural biological process. The fertilization requires activated sperm to go into the ooplasm and induce the oocyte to form a pronucleus. The important steps involved in the success of fertilization by ICSI are immobilization of sperm and deliver that sperm into the ooplasm in optimal position. ${ }^{8}$ The sperm immobilization by crushing the tail can damage plasma membrane and initiate gradual breakage of sperm membrane in other part of which induce the sperm factor, phospholipase $\mathrm{C}$ zeta (PLC-zeta, PLC $\zeta$ ), and then trigger cytosolic $\mathrm{Ca} 2+$ oscillations to elicit oocyte activation and early embryo development up to the blastocyst stage., $3,9-11$ Simultaneously, the sperm nucleus decondensing factor (SDF) from the oocyte can pass through the sperm membrane disruption which causes the sperm head swelling and rupture of its plasma membrane that increasing the release of sperm factor in which localized in the perinuclear theca of the sperm head and promote oocyte activation in another way and finally result in successful fertilization. ${ }^{12,13}$ The deposition of entire immobilized sperm in the oocyte is a crucial step. Therefore, the direction of the sperm inserted into the oocyte (headfirst and tail-first) may not be an important matter. However, the sperm head is relatively larger and more visible than the tail. During ICSI, the headfirst aspiration of the sperm into the injection micropipette is easier and takes less time then injected tail-first into the oocyte. On the other hand, aspiration of sperm tail first into the micropipette is more difficult and takes a longer time then injected headfirst into the oocyte. The exposure of the oocyte during ICSI to an unfavorable environment i.e. HEPES buffered medium, room temperature and the air outside the incubator might cause some possible adverse effects to the fertilization capacity of the oocyte. ${ }^{14}$ Hiraoka et al, mentioned that there was a trend toward low fertilization in headfirst ICSI, but of insignificant difference, because more time was consumed and such findings were similar to this study. ${ }^{7}$ However, further study is required to elucidate this interesting issue in the future.

In a previous report, Suh et al, revealed no significant difference in blastocyst rates of headfirst and tail-first ICSI (44.4\% versus 50.0\%). ${ }^{5}$ Similarly, Woodward et al, had equal pregnancy rates between the two procedures and piezo ICSI of headfirst and tail-first injections also gave a comparable clinical pregnancy rate $(33.2 \%$ versus $30.9 \%$ ) which were consistent with the outcome of this study. ${ }^{6,7}$

\section{CONCLUSION}

The results showed that the direction of headfirst and tailfirst sperm injections had no significant effect on oocyte survival, fertilization, blastocyst development, implantation, and clinical pregnancy. The successful deposition of sperm into the ooplasm is the crucial step for fertilization. The findings may be of benefit to maximize the outcomes of ICSI.

\section{ACKNOWLEDGMENTS}

The author would like to thank the patients and personnel in the Department of Obstetrics and Gynecology, Buddhachinaraj Hospital Medical School for their kind cooperation and the professional English reviewer, $\mathrm{Mr}$. Robert Moore, for his kind help in editing this manuscript.

\section{Funding: No funding sources}

Conflict of interest: None declared

Ethical approval: The study was approved by the Institutional Ethics Committee

\section{REFERENCES}

1. Okabe M. The cell biology of mammalian fertilization. Develop. 2013;140:4471-9.

2. Talbot P, Shur BD, Myles DG. Cell adhesion and fertilization: Steps in oocyte transport, sperm-zona pellucida interactions, and sperm-egg fusion. Biol Reprod. 2003;81:1-7.

3. Neria QV, Leea B, Rosenwaksa Z, Machacab K, Palermo GD. Understanding fertilization through intracytoplasmic sperm injection (ICSI). Cell Calcium. 2014;55:24-37.

4. Simopoulou M, Giannelou P, Bakas P. Making ICSI safer and more effective: a review of the human oocyte and ICSI practice. In Vivo. 2016;30:387-400.

5. Suh TK, Moon JS, Kim KC, Lee TH, Chun SS. Effect of head or tail first sperm injection into oocytes in intracytoplasmic sperm injection. Fertil Steril. 1997;68:S152.

6. Woodward BJ, Campbell KH, Ramsewak SS. A comparison of headfirst and tailfirst microinjection of sperm at intracytoplasmic sperm injection. Fertil Steril. 2008;89:711-4.

7. Hiraoka K, Kitamura S, Otsuka Y, Kawai K, Harada $\mathrm{T}$, Ishikawa $\mathrm{T}$. Effects of sperm direction in PiezoICSI on oocyte survival, fertilization, embryo development and implantation ability in humans: A preliminary study. J Obstet Gynecol Res. 2018;44:1963-9.

8. Yanagida $\mathrm{K}$, Katayose $\mathrm{H}$, Hirata S, Yazawa $\mathrm{H}$, Hayashi S, Sato A. Influence of sperm immobilization on onset of $\mathrm{Ca}+2$ oscillations after ICSI. Hum Reprod. 2001;16:148-52.

9. Swann K. A cytosolic sperm factor stimulates repetitive calcium increases and mimics fertilization in hamster eggs. Develop. 1990;110:1295-302. 
10. Dozortsev D, Rybouchkin A, De Sutter P, Dhont M. Sperm plasma membrane damage prior to intracytoplasmic sperm injection: a necessary condition for sperm nucleus decondensation. Hum Reprod. 1995;10:2960-4.

11. Nomikos M, Kashir J, Lai FA. The role and mechanism of action of sperm PLC-zeta in mammalian fertilisation. Biochem J. 2017;474:365973.

12. Dozortsev D, Qian C, Ermilov A, Rybouchkin A, De Sutter P, Dhont M. Sperm-associated oocyteactivating factor is released from the spermatozoon within 30 min after injection as a result of the spermoocyte interaction. Hum Reprod. 1997;12:2792-6.
13. Caglar GS, Hammadeh M, Asimakopoulos B, Nikolettos N, Diedrich K, Al-Hassani S. In vivo and in vitro decondensation of human sperm and assisted reproduction technologies. In Vivo. 2005;19:623-30.

14. Swain JE, Carrell D, Cobo A, Meseguer M, Rubio C, Smith GD. Optimizing the culture environment and embryo manipulation to help maintain embryo developmental potential. Fertil Steril. 2016;105:57187.

Cite this article as: Pongsuthirak P. Comparison between headfirst and tail-first direction of sperm for intracytoplasmic sperm injection. Int J Reprod Contracept Obstet Gynecol 2019;8:4177-81. 